\title{
A method for Kinematic Calibration of a Parallel Robot by using one camera in hand and a spherical object
}

\author{
Alberto Traslosheros, José María Sebastián, Eduardo Castillo, Flavio Roberti, Ricardo Carelli
}

\begin{abstract}
The main purpose of robot calibration is the correction of the possible errors in the robot parameters. This paper presents a method for a kinematic calibration of a parallel robot that is equipped with one camera in hand. In order to preserve the mechanical configuration of the robot, the camera is utilized to acquire incremental positions of the end effector from a spherical object that is fixed in the word reference frame. The positions of the end effector are related to incremental positions of resolvers of the motors of the robot, and a kinematic model of the robot is used to find a new group of parameters which minimizes errors in the kinematic equations. Additionally, properties of the spherical object and intrinsic camera parameters are utilized to model the projection of the object in the image and improving spatial measurements. Finally, the robotic system is designed to carry out tracking tasks and the calibration of the robot is validated by means of integrating the errors of the visual controller.
\end{abstract}

\section{INTRODUCTION}

A CCURACY is a crucially important performance specification for parallel kinematic machines (PKM), particularly for those that are involved in tasks as can be surgery, material machining, electronics manufactory, products assembling, among other. In this work calibration method is used to improve a widely studied image based visual controller [1]. In contrast to serial robots, parallel robots (or parallel kinematic machines) are characterized by high structural stiffness, high load operation, high speed and acceleration of the end effector and, high accuracy for end effector positioning. This accuracy, however, relies on a robust and accurate calibration, which is a difficult problem both from a theoretical and a practical point of view, even if it may be performed off-line. Robot accuracy can be affected by increasing backlash due to robot operation, thermal effects, elements deformation [2], robot control and manufacturing errors. In order to calibrate a robot, there exist a wide variety of methods (even hybrid calibration

Manuscript received March 15, 2011. This work was supported in part by the Universidad Politécnica de Madrid and International Cooperation Spanish Agency (AECI), A/026481/09.

A. Traslosheros and J. M. Sebastián are with the Technical University of Madrid, DISAM, José Gutierrez Abascal 2, CO 28003 Spain. Phone (+34) 913363 061, email: atraslosheros@etsii.upm.es and jsebas@etsii.upm.es. E. Castillo is with the National Technical Institute of Mexico, Departament of Mechatronics, Cerro Blanco No. 141., CO 76090, Queretaro, Mexico. Phone (+52) 442229 0804, email: ecastilloca@ipn.mx

F. Roberti and R. Carelli are with the National University of San Juan, Av. San Martín Oeste 1109, CO 5400 Argentina. Phone (+54) 02644213 303, email: froberti@inaut.unsj.edu.ar and rcarelli@inaut.unsj.edu.ar. Department of Automatics, National University of San Juan, Argentina. methods), but in general, it is possible to classify different calibration strategies in three main groups (by considering the location of measurement instruments and its additional elements): External calibration, it is based on measurements of the positions of the end effector (or other structural element) of the robot by means of an external instrument. Constrained calibration groups those methods that rely on mechanical elements in order to constraint some kind of motion of the robot during the calibration process, this method is generally simple and it is considered the most inexpensive. And finally, auto calibration that consist on that methods that calibrates the robot automatically and even during the robot operation [3], in general auto (or self) calibration method is more expensive due to its complexity and even it includes redundant sensors [4] or elements. External calibration can be done by measuring completely or partially the pose parameters of the platform. Measurements of the pose of a platform can be done with a laser and a coordinate measuring machine (CMM)[5], commercial visual systems (optical system and infrared light) [6-8], visual systems and microscopes [9], laser sensor [10], by adding passive legs [11] or by constraining elements [12], with a interferometer [13], with a LVDT and inclinometers in [12,14], theodolite [15], with gauges [16], with double ball bar (DBB) $[17,18]$, by inspecting a machined part that is dedicated to the calibration process [19], accelerometers [20], or with visual systems and patterns widely studied (chess-board and similar patterns) [21-23]. Above examples are different strategies that obtain kinematic information of the robot, but in general, calibration methods impose virtual or real constraints on the poses of the end effector (or mobile elements). It is important to bring out that by choosing the appropriate method, a kinematic calibration can be an economical and practical technique for improve accuracy of a PKM [24].

Generally speaking, the principle of the calibration process is to get the constraints at a large enough number of measured poses (called calibration poses) in order to conclude which is the best geometry (distances or angles of elements) of the robot that satisfies them. The basic idea it has been applied to calibration of serial and parallel robots and the main difference has been the measurement instruments and strategies. Visual methods are becoming more popular due to its simplicity and because it can be an inexpensive method in comparison to others, for parallel robots it was first proposed by Amirat [25]. Visual methods that propose a monocular system [21-23] propose to utilize a 
pattern (marks on a flat surface), these kind of patterns are employed in camera calibration, where is possible to obtain intrinsic and extrinsic parameters [26]. By means of the extrinsic parameters of the camera it is possible (by attaching the camera or the pattern to the rend effector of the robot) to obtain the pose of the end effector of the robot and consequently those poses can be considered as calibration poses.

In this paper an inexpensive and external calibration method is proposed to calibrate a parallel robot of 3 DOF. As was mentioned, the proposed method uses incremental positions from the resolvers of the motors and visual information that is obtained from a spherical element (in order to obtain incremental positions of the end effector). Once the above information is obtained, then it is replaced in the constraint equations in order to solve them numerically and obtaining the best set of geometrical parameters that satisfies the set of equations. Finally, in order to test the calibration method, the parameters obtained are used in a visual servoing controller (image based) and results are compared to the controller that uses nominal parameters.

\section{THE SyStem RoBotenIS AND ITS COMPONENTS}

The main objective of proposed method it is to calibrate the parallel robot of the system called: Robotenis (Fig. 1 a). A calibration of the robot is required in order to improve its accuracy in visual servoing algorithms and develop future tasks as can be the Ping-Pong game.

The system Robotenis was designed in order to study and design visual servoing controllers and to carry out object tracking under dynamic environments. The mechanical structure of system Robotenis is inspired by the DELTA robot [27][28] and its vision system is based in one camera that is allocated at the end effector of the robot. Basically, the platform Robotenis (Fig. 1 a) consists of a parallel robot and a visual system for acquisition and analysis. The maximum end effector speed of the parallel robot is $6 \mathrm{~m} / \mathrm{s}$ and its maximum acceleration is $58 \mathrm{~m} / \mathrm{s}^{2}$. The visual system of the platform Robotenis [29] is composed of a 50 grams camera that is located at the end effector (Fig. $1 \mathrm{~b}$ ) and a frame grabber (SONY XCHR50 and Matrox Meteor 2$\mathrm{MC} / 4$ respectively). The motion system is composed of three AC brushless servomotors, Ac drivers (Unidrive), planetary gearboxes (10:1) and the joint controller is implemented in a DS1103 card (implemented in ANSI C).

\section{CONSTRAInT EQuATIONS}

Constraint equations that are used in the calibration method were obtained from a kinematic model of the robot [30]. In the application of the method, it is important to bring out that in the constraint equations, transformation matrix from the camera to the end effector is not considered and it is obtained by an additional method that is not shown in the present work. Thus, transformation matrix between the camera and the end effector was divided in its rotation and translation, rotation matrix was obtained independently to the robot calibration, on the other hand translation matrix is not necessary, because the camera is fixed to the end effector and the model of the constraint equations is an incremental one. In the Fig. 2 it is shown a sketch of the parallel robot, where $\Sigma_{\mathrm{O}}$ is the robot reference frame, $\mathrm{a}_{\mathrm{i}}$ are arm lengths, $\mathrm{b}_{\mathrm{i}}$ are forearm lengths, arms and forearms are connected in $\boldsymbol{B}_{\boldsymbol{i}}$, $H_{i}$ are distances from the robot reference frame $\left(\boldsymbol{O}_{\boldsymbol{x y z}}\right)$ to the revolute axis of motors $\left(\boldsymbol{A}_{\boldsymbol{i}}\right), h_{i}$ are the distances from the center of the end effector $(\boldsymbol{P})$ to the forearm $\left(\boldsymbol{C}_{\boldsymbol{i}}\right), \theta_{i}$ are the motor angles from a home position (unknown), $\phi_{i}$ is the angle in which point $\boldsymbol{A}_{\boldsymbol{i}}$ is allocated in the plane $X Y$ of the robot frame reference and $i=1,2,3$.

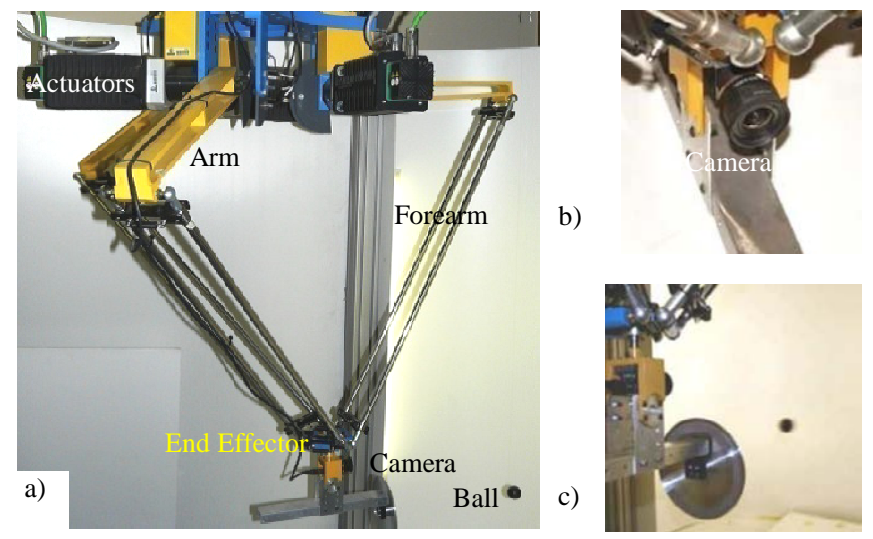

Fig. 1 a) System Robotenis, b) Robot camera, c) Environment

Constraint equations are based on the movement of the forearms respect to the robot arms [30]. In the Delta robot, forearms are parallelograms that link the end effector to the arms by means of spherical joints. Spherical joints allow modeling the movement of the end effector as the intersection of three spherical surfaces that are described by points $C_{i}$ in Fig. 3. By inspecting Fig. 2 and Fig. 3 it is possible to obtain the surface of a sphere described by the point $\boldsymbol{C}_{\boldsymbol{i}}$ with center in $\boldsymbol{B}_{\boldsymbol{i}}$ as:

$\Gamma_{i}=\left(C_{i x}-B_{i x}\right)^{2}+\left(C_{i y}-B_{i y}\right)^{2}+\left(C_{i z}-B_{i z}\right)^{2}-b_{i}^{2}=0$

, where $\boldsymbol{B}_{\boldsymbol{i}}$ and $\boldsymbol{C}_{\boldsymbol{i}}$ can be expressed in the robot frame as:

$$
\begin{aligned}
{ }^{\Sigma_{o}}\left[\begin{array}{l}
B_{x} \\
B_{y} \\
B_{z}
\end{array}\right]_{i} & =\left[\begin{array}{c}
(H+a c \theta) c \phi \\
(H+a c \theta) s \phi \\
a s \theta
\end{array}\right]_{i} \\
{ }^{\Sigma_{o}}\left[\begin{array}{l}
C_{x} \\
C_{y} \\
C_{z}
\end{array}\right]_{i} & =\left[\begin{array}{l}
P_{x} \\
P_{y} \\
P_{z}
\end{array}\right]-\left[\begin{array}{ccc}
c \phi & -s \phi & 0 \\
s \phi & c \phi & 0 \\
0 & 0 & 1
\end{array}\right]_{i}\left[\begin{array}{c}
-h \\
0 \\
0
\end{array}\right]_{i}
\end{aligned}
$$

Note that in above equations $c \theta_{i}=\cos \theta_{i}, s \theta_{i}=\sin \theta_{i}$, $\boldsymbol{P}=\left[P_{x}, P_{y}, P_{z}\right]^{\prime}, \boldsymbol{B}_{\boldsymbol{i}}=\left[B_{x}, B_{y}, B_{z}\right]_{i}^{\prime}$, etc. On the other hand by supposing that we can obtain incremental joint and end effector positions we have that each absolute measurement can be expressed as:

$\theta_{i k}=\theta_{i 0}+\Theta_{i k} \quad$ and $\quad \boldsymbol{P}_{k}=\boldsymbol{P}_{0}+\Pi_{\mathrm{k}}$ 
Observe that $\theta_{i 0}$ and $\boldsymbol{P}_{\mathbf{0}}$ are unknown and, $\Theta_{i k}=\Delta \theta_{i k}=$ $\theta_{i k}-\theta_{i(0)}$ and $\boldsymbol{\Pi}_{\mathrm{k}}=\Delta \boldsymbol{P}_{k}=\boldsymbol{P}_{k}-\boldsymbol{P}_{0}$ are incremental measuring (obtained from resolvers and camera): $\mathbf{V}_{\mathbf{k}}=\left[\Theta_{1}, \Theta_{2}, \Theta_{3}, \Pi_{x}, \Pi_{y}, \Pi_{z}\right]_{k}$, where $\Pi_{\mathbf{k}}$ are measured and are parameters that are expressed as incremental positions of the end effector and they are measured from sphere images (section $\mathrm{V}$ ), $k$ is the incremental measurement number.

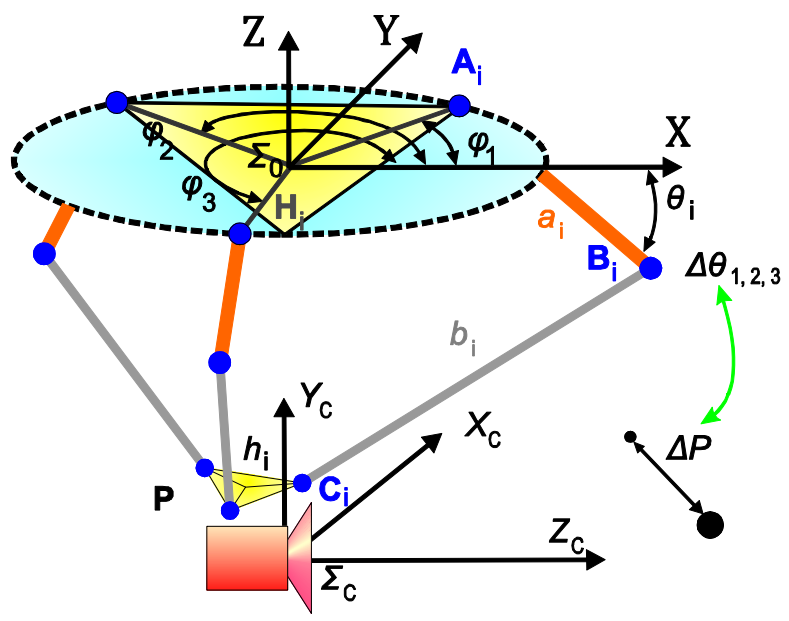

Fig. 2 Sketch of the system Robotenis.

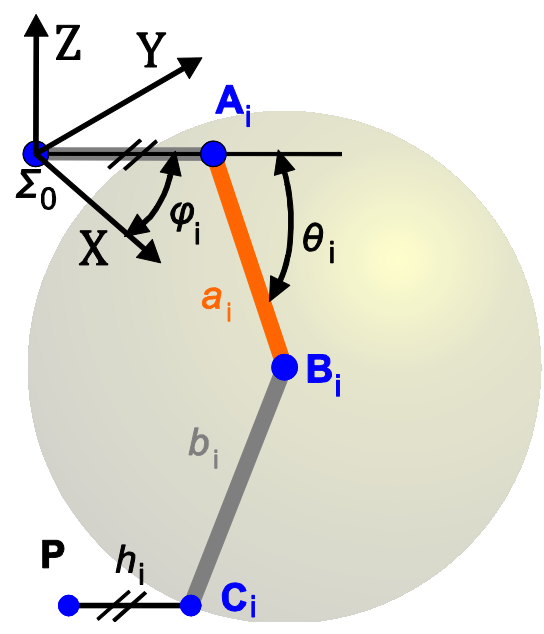

Fig. 3 Sketch of one leg of the system Robotenis.

\section{CONSTRAint EQuATIONS AND ThEM SOLUTION}

Not all parameters of the robot have effect on the kinematic model and as a consequence they cannot be identified, this is known as observability. An observability measure can be derived from the Jacobian of the constraint equations. This Jacobian is called the observation matrix and observable parameters can be obtained by its QR decomposition [31]. In our model by means of the $\mathbf{Q R}$ decomposition influence of the parameters $\phi_{i}$ and $H_{i}$ are difficult to observe in the model, for this reason they are not identified in this work and their nominal value is taken into account. On the other hand identifiable parameters are: $a_{i}, b_{i}, h_{i}$ and reference position of the actuators and the end effector of the robot: $\theta_{i 0}$ and $\boldsymbol{P}_{\mathbf{0}}$ respectively ( $i=1,2,3$ and $\boldsymbol{P}_{\mathbf{0}}$ is the initial end effector position). Thus unknown parameters can be expressed as: $\boldsymbol{u}_{\boldsymbol{i}}=\left[a_{i}, b_{i}, h_{i}, \theta_{i 0}\right]$ and $\mathbf{U}=\left[u_{1} u_{2} u_{3} P_{x 0} P_{y 0} P_{z 0}\right]$.
And substituting incremental equations (3) in (2) and (1), then constraint equations can be arranged as:

$$
\begin{aligned}
\Gamma_{i}= & \left(\left(a_{i} c\left(\theta_{i}+\Theta_{i k}\right)+H_{i}\right) c \phi_{i}-P_{x}-h_{i} c \phi_{i}-\Pi_{x k}\right)^{2} \\
& +\left(\left(a_{i} c\left(\theta_{i}+\Theta_{i k}\right)+H_{i}\right) s \phi_{i}-P_{y}-h_{i} s \phi_{i}-\Pi_{y k}\right)^{2} \\
& +\left(a_{i} s\left(\theta_{i}+\Theta_{i k}\right)-P_{z}-\Pi_{z k}\right)^{2}-b_{i}^{2}=0
\end{aligned}
$$

In order to solve, equations in (4) are grouped as:

$\Phi\left(\mathbf{U}, \mathbf{V}_{\mathrm{k}}\right)=\left[\Gamma_{11}, \ldots, \Gamma_{1 k}, \Gamma_{21}, \ldots, \Gamma_{2 k}, \Gamma_{31}, \ldots, \Gamma_{3 k}\right]^{\prime}$

Note that constraint equations are not linear, its solution is no exactly satisfied thus commonly a nearly exact solution is obtained by numerical algorithms such as Gauss-Newton. Expressing constraints (5) in them Taylor linear approximation in order to solve iteratively.

$\Phi\left(\mathbf{U}_{\mathrm{n}+1}, \mathbf{V}_{\mathrm{k}}\right) \approx \boldsymbol{\Phi}\left(\mathbf{U}_{\mathrm{n}}, \mathbf{V}_{\mathrm{k}}\right)+J\left(\mathbf{U}_{\mathrm{n}}, \mathbf{V}_{\mathrm{k}}\right)\left(\mathbf{U}_{\mathrm{n}+1}-\mathbf{U}_{\mathrm{n}}\right)=0$

, where $n$ is the iteration number and $J_{3 k \times 18}$ is the Jacobian of the constraint equations or the observation matrix that it is given by:

$J\left(\mathbf{U}_{\mathrm{n}}, \mathbf{V}_{\mathrm{k}}\right)=\left[\begin{array}{c}J_{1 k} \\ J_{2 k} \\ J_{3 k}\end{array}\right]$

$J_{1 k}=\left[\begin{array}{c}\frac{\partial \Gamma_{11}}{\partial a_{1}} \ldots \frac{\partial \Gamma_{11}}{\partial \theta_{10}}, 0, \ldots, 0,0, \ldots, 0, \frac{\partial \Gamma_{11}}{\partial P_{x 0}}, \frac{\partial \Gamma_{11}}{\partial P_{y 0}}, \frac{\partial \Gamma_{11}}{\partial P_{z 0}} \\ \vdots \\ \frac{\partial \Gamma_{1 k}}{\partial a_{1}} \ldots \frac{\partial \Gamma_{1 k}}{\partial \theta_{10}}, 0, \ldots, 0,0, \ldots, 0, \frac{\partial \Gamma_{1 k}}{\partial P_{x 0}}, \frac{\partial \Gamma_{1 k}}{\partial P_{y 0}}, \frac{\partial \Gamma_{1 k}}{\partial P_{z 0}}\end{array}\right]$

$J_{2 k}=\left[\begin{array}{c}0, \ldots, 0, \frac{\partial \Gamma_{21}}{\partial a_{2}} \ldots \frac{\partial \Gamma_{21}}{\partial \theta_{20}}, 0, \ldots, 0, \frac{\partial \Gamma_{21}}{\partial P_{x 0}}, \frac{\partial \Gamma_{21}}{\partial P_{y 0}}, \frac{\partial \Gamma_{21}}{\partial P_{z 0}} \\ \vdots \\ 0, \ldots, 0, \frac{\partial \Gamma_{2 k}}{\partial a_{2}} \ldots \frac{\partial \Gamma_{2 k}}{\partial \theta_{20}}, 0, \ldots, 0, \frac{\partial \Gamma_{2 k}}{\partial P_{x 0}}, \frac{\partial \Gamma_{2 k}}{\partial P_{y 0}}, \frac{\partial \Gamma_{2 k}}{\partial P_{z 0}}\end{array}\right]$

$J_{3 k}=\left[\begin{array}{c}0, \ldots, 0,0, \ldots, 0, \frac{\partial \Gamma_{31}}{\partial a_{3}} \ldots \frac{\partial \Gamma_{31}}{\partial \theta_{30}}, \frac{\partial \Gamma_{31}}{\partial P_{x 0}}, \frac{\partial \Gamma_{31}}{\partial P_{y 0}}, \frac{\partial \Gamma_{31}}{\partial P_{z 0}} \\ \vdots \\ 0, \ldots, 0,0, \ldots, 0, \frac{\partial \Gamma_{3 k}}{\partial a_{3}} \ldots \frac{\partial \Gamma_{3 k}}{\partial \theta_{30}}, \frac{\partial \Gamma_{3 k}}{\partial P_{x 0}}, \frac{\partial \Gamma_{3 k}}{\partial P_{y 0}}, \frac{\partial \Gamma_{3 k}}{\partial P_{z 0}}\end{array}\right]$

Finally, parameters are iteratively obtained by:

$\mathbf{U}_{\mathrm{n}+1}=\mathbf{U}_{\mathrm{n}}-J\left(\mathbf{U}_{\mathrm{n}}, \mathbf{V}_{\mathrm{k}}\right)^{+} \boldsymbol{\Phi}\left(\mathbf{U}_{\mathrm{n}}, \mathbf{V}_{\mathrm{k}}\right)$

, where $J\left(\mathbf{U}_{\mathrm{n}}, \mathbf{V}_{\mathrm{k}}\right)^{+}$is the pseudoinverse of the Jacobian of the system of equations. Observe that observability analysis and pose selection are done in order to obtain a valid pseudoinverse of the Jacobian. The numerical method requires initial values $\left(\mathbf{U}_{0}\right)$ that are given by the nominal parameters and a pose of the robot (given from nominal parameters).

\section{The MEASUREMENT System}

The measurement system mainly consists of a fixed ball (hanging by a thread) and a calibrated camera that it is allocated on the end effector of the robot. The measurement method registers, on the one hand, the initial position of the 
end effector, and on the other hand, the initial positions of resolvers. Thus, once incremental positions are measured, both measures are related through constraint equations to solve unknown parameters.

\section{A. Selection of Calibration Poses}

In order to solve the system of constraint equations, a set of incremental (resolvers and end effector) poses is needed, and simulations shows that 6 different poses are at least required (perfect measurements and perfect system). However perfect measurements do not exist and is necessary to choose best calibration poses. There exist approaches [4,32], where a noise amplification index is used to identify poses in which errors in the parameters of the robot are especially critical; this is given when a calibration pose is near from a singularity or from workspace boundaries. However in a practical calibration process, if poses are extremely near of singularities, those poses are able to produce unpredictable movements (due to kinematic errors). In this work, the robot workspace is numerically well known (by using nominal values) and the calibration poses are chosen randomly from the workspace boundaries, Fig. 4. Thus if a pose is between 5 and $10 \mathrm{~cm}$ away from a singularity (and boundaries of the worspace), then the pose is included as a calibration pose.



Fig. 4 Selection of the calibration poses (off-line), which are chosen between 5 and $10 \mathrm{~cm}$ far from singularities and boundaries of the workspace (the workspace of the robot has hexagonal shape due to limitations of the passive joins of the parallelograms of the robot).

Experiments were implemented with 5000 of poses and each measure is the mean of 100 images in order to avoid noise from image acquisition (image acquisition rate: 120 FPS, $8.34 \mathrm{~ms}$ ). Due to its simplicity, calibration process is done automatically in 70 minutes and it is possible to calibrate on-line. On-line calibration could be implemented in a pick and place tasks, but requires having a camera allocated on the end effector meanwhile the ball is observed and images are taken when the robot stops moving.

Additionally it has been mentioned that a spherical object has been chosen as reference of the end effector and some considerations of the spherical object and its projection on the image plane has to be taken into account, Fig. 5. Each measurement is done in two steps, first a simplified model is considered as in Fig. 6, where two sub-pixel points are acquired from the image (distortion is previously corrected), secondly a correction of measurements is done by considering elliptical projection of the ball. Additionally, it must take into account that the method depends on: the knowledge of the parameters of the camera calibration, as is described in [33], and the knowledge of the size of a fixed ball.

\section{B. Simplified Ball Projection Model}

Objective of this method is to obtain the position of the center of a spherical object. Position is obtained from the projection of the ball in the plane of the image. The method takes into account that the projected ball does not correspond to the real center and diameter of the ball. Proposed method is iterative and a first approximation is obtained by a simplified 2D model as shown in Fig. 6. Model in Fig. 6 is contained in a plane in the coordinates $X$ and $Z$ that are in the camera coordinate frame $\left(X_{c}, Y_{c}, Z_{c}\right)$. On the other hand points that are in the image are related by a line that is tangent to the sphere and passes through optical center of the camera, that is:

$X=\alpha_{1} Z \quad$ and $\quad X=\alpha_{2} Z$

Sphere is fixed in the space, its diameter is known (38mm) and the distance of the line that is tangent to the perimeter to the center of the sphere $\left(L_{B}, C_{B}\right)$ are related by:

$R_{B}=\frac{L_{B}-\alpha C_{B}}{\sqrt{1+\alpha^{2}}}$

, where $R_{B}=19 \mathrm{~mm}$ and $\alpha$ can be cleared from eq. (10):

$\alpha_{1,2}=\frac{L_{B} C_{B} \pm \sqrt{L_{B}^{2} C_{B}^{2}-\left(C_{B}^{2}-R_{B}^{2}\right)\left(L_{B}^{2}-R_{B}^{2}\right)}}{C_{B}^{2}-R_{B}^{2}}$

,where $C_{B}$ is supposed positive and $C_{B}>R_{B}$, otherwise the ball cannot be seen. By substituting eq. (10) and (9) in the following circumference equation from Fig. 6:

$\left(\alpha_{1,2} Z-L_{B}\right)^{2}+\left(Z-C_{B}\right)^{2}=R_{B}^{2}$

Clearing for $Z$ and simplifying it is possible to obtain:

$Z_{1,2}=\frac{\alpha_{1,2} L_{B}+C_{B}}{\alpha_{1,2}^{2}+1}$

Thus the purpose is to obtain the center of the ball $\left(L_{B}, C_{B}\right)$ from the known points in the image $\left(x_{u 1}, x_{u 2}\right)$. For points that are tangent to the sphere it is easy to see that:

$X_{1} / Z_{1}=x_{u 1} / f ; \quad X_{2} / Z_{2}=x_{u 2} / f$ 
,where $f$ is the focal distance. The algorithm that is implemented is iterative and its solution is obtained in a few iterations. Initial solution is obtained supposing that:

$Z_{1} \approx Z_{2} \approx C_{b} ;\left|X_{2}-X_{1}\right|=2 R_{B}$

In equation (11) parameters $\mathrm{L}_{\mathrm{B}}$ and $\mathrm{C}_{\mathrm{B}}$ are the position of the center $\left(X_{B}=L_{B}\right.$ and $\left.Z_{B}=C_{B}\right)$ of the circle in Fig. 6 and they are unknown. At this point, must be cleared that the algorithm is iterative and it needs an initial position that is given by:

$C_{B}=\frac{2 f R_{B}}{\left|x_{u 2}-x_{u 1}\right|}$ and $\quad L_{B}=\frac{x_{u 2}+x_{u 1}}{2 f} C_{B}$

Values obtained in eq. (16) and equations (11), (13) and (14) are iteratively utilized to obtain $C_{B}$ and $L_{B}$ that are repeatedly substituted in eq. (12) until there are not differences between old and new values.



Fig. 5 Ball projection in the image plane.

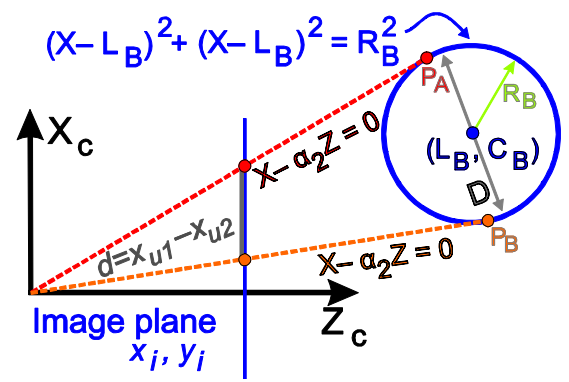

Fig. 6 Ball projection in the image plane.

\section{A. Ellipse Ball Projection in Z correction}

One condition of the method is that the spherical object must be fully visible. This condition guarantees that the projection of the sphere is an ellipse Fig. 7 (or a circumference if the projection lies on the center of the image). Note in Fig. 6 that $x_{u 1}$ and $x_{u 2}$ are not on the axis of the projected ellipse, thus the diameter considered in the calculus of $\mathrm{Z}$ contains a small error due to the projected ball.

In order to correct the diameter that is considered in the calculus of $\mathrm{Z}$, the minor axis of the ellipse has to be calculated. Once the minor axis is calculated then $2 b_{e}$ is replaced in (16) instead of $\left|x_{u 2}-x_{u 1}\right|$, thus the ball position is calculated by earlier method for a second time. This second step mainly depends on angle $\theta_{z}$ and the method stops when $\theta_{z}$ is constant or almost constant (usually two or three cycles). $\theta_{z}$ is the angle between the optical axis and the line that passes through the center of the ball and the center of the projected ball. Note that $\theta_{z}$ coincides with the angle that the image plane can be rotated around the minor axis of the ellipse in order to project a circle on the plane image, thus:

$\theta_{z}=\operatorname{acos}\left(\frac{Z}{\sqrt{\left(X^{2}+Y^{2}+Z^{2}\right.}}\right)$

The minor $\left(b_{e}\right)$ and major $\left(a_{e}\right)$ axes of the projected ellipse are related by:

$b_{e}=a_{e} \cos \left(\theta_{z}\right)$

The projected ellipse in its rotated canonical parametric form can be expressed as:

$x_{u e}=b_{e} \cos \left(\theta_{e}\right) \cos \left(\theta_{r}\right)-a_{e} \sin \left(\theta_{e}\right) \sin \left(\theta_{r}\right)+x_{u c}$

$y_{u e}=b_{e} \cos \left(\theta_{e}\right) \sin \left(\theta_{r}\right)+a_{e} \sin \left(\theta_{e}\right) \cos \left(\theta_{r}\right)+y_{u c}$

,where $\theta_{e}$ is the angle of a point in the canonical form of the ellipse, $\theta_{r}$ is the angle that the ellipse is rotated around the $\mathrm{Z}$ axis of the camera (eq. (20)) and $\left(x_{u c}, y_{u c}\right)$ is the center of the ellipse in the image, Fig. 8.

$\theta_{r}=\operatorname{atan}\left(-\frac{y_{u c}}{x_{u c}}\right)$

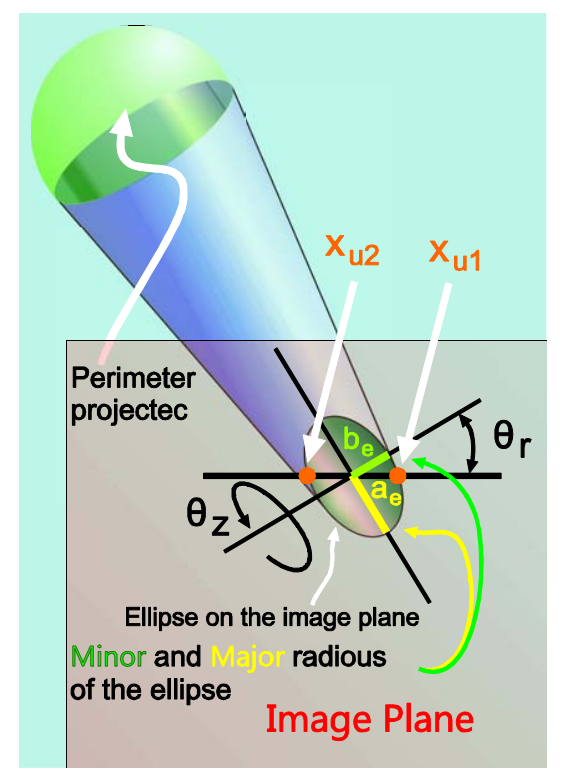

Fig. 7 Ellipse of the sphere projection on the image plane.

On the other hand we know that $\left(x_{u 1}, y_{u 1}\right)$ and $\left(x_{u 2}, y_{u 2}\right)$ belong to the ellipse, and by simple inspection in Fig. 7: $x_{u c}-x_{u e}=\left(x_{u 2}-x_{u 1}\right) / 2, \quad y_{u c}-y_{u e}=\left(y_{u 2}-y_{u 1}\right) /$ $2=0$ and substituting in (19) we can obtain that:

$\left(x_{u 2}-x_{u 1}\right) / 2=b_{e} \cos \left(\theta_{e}\right) \cos \left(\theta_{r}\right)-a_{e} \sin \left(\theta_{e}\right) \sin \left(\theta_{r}\right)$

$0=b_{e} \cos \left(\theta_{e}\right) \sin \left(\theta_{r}\right)+a_{e} \sin \left(\theta_{e}\right) \cos \left(\theta_{r}\right)$ 
Substituting (18) in (21) and clearing for $a_{e}$ and $\theta_{e}$ finally:

$a_{e}=\left|\frac{\left(x_{u 2}-x_{u 1}\right) / 2}{\cos \left(\theta_{z}\right) \cos \left(\theta_{e}\right) \cos \left(\theta_{r}\right)-\sin \left(\theta_{e}\right) \sin \left(\theta_{r}\right)}\right|$

$\theta_{e}=\operatorname{atan}\left(-\tan \left(\theta_{r}\right) \cos \left(\theta_{z}\right)\right)$

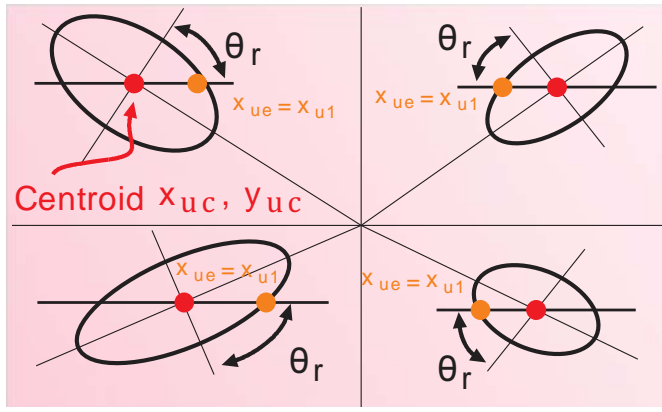

Fig. 8 Angle $\theta_{r}$.

\section{RESULTS}

In order to test the calibration method, nominal parameters and calibrated parameters are compared when they are utilized in an image based visual controller. The robot and its visual controller are designed with the purpose of carry out tracking tasks, [34]. Visual controller is based in a well established architecture called: dynamic image-based look-and-move visual servoing. In this scheme visual controller makes use of image estimates (position, velocity), Jacobian of the robot and its kinematic model in order to act over the robot actuators. Thus errors in the kinematic model generate undesirable movements that have an effect on the performance of the visual controller. In this section we compare the performance of the visual controller of the robot when the controller makes use of the nominal kinematic parameters and calibrated kinematic parameters (shown in TABLE 1). In order to compare the visual controller, a tracking index performance (TIP) is defined as follows:

$T I P=\frac{\sum_{k=1}^{N}|e(k)|}{\sum_{k=1}^{N} T_{k}}$

Visual controller is based in velocity estimation and tracking error of the object. This index is used to measure the error of each test. Particularly in this section $k$ means the visual controller sample, $|e(k)|$ is the norm of the tracking error in the instant $k, T_{k}$ is the time in the instant $k$.

Both parameters collections, calibrated and not calibrated, are considered into the robot kinematics in order to test the visual controller of the robot. Controller performance is obtained by means of index in eq. (23) (TIP index), results of 400 tests (mean of 100 tests per kind of movement and kinematic parameters) are shown in TABLE 2, where two type of movements were implemented: a static test, where the ball remains static and the relative reference between the camera end end-effector is fixed for a long time, and a dynamic test, where relative reference is shifting every sample time. Indexes show how the error of the visual controller increases although kinematic errors are small in comparison to the nominal parameters.

TABLE 1 Robot kinematic parameters, nominal and calibrated

\begin{tabular}{|l|c|c|c|c|c|c|}
\hline \hline & \multicolumn{3}{|c|}{ Nominal (lengths in mm) } & \multicolumn{3}{c|}{ Calibrated (lengths in mm) } \\
\cline { 2 - 7 } & $i=1$ & $i=2$ & $i=3$ & $i=1$ & $i=2$ & $i=3$ \\
\hline$a_{i}$ & 500 & 500 & 500 & 500.89 & 500.01 & 499.96 \\
\hline$b_{i}$ & 1000 & 1000 & 1000 & 1002.86 & 1001.37 & 1001.28 \\
\hline$h_{i}$ & 50 & 50 & 50 & 48.69 & 49.96 & 48.68 \\
\hline$\theta_{i}$ & $0^{\circ}$ & $0^{\circ}$ & $0^{\circ}$ & $0.3156^{\circ}$ & $0.8440^{\circ}$ & $0.9362^{\circ}$ \\
\hline$P_{x}$ & \multicolumn{3}{|c|}{0} & \multicolumn{3}{c|}{5.3104} \\
\hline$P_{y}$ & \multicolumn{3}{|c|}{0.3166} \\
\hline$P_{z}$ & 0 & \multicolumn{3}{c|}{743.7405} \\
\hline$\phi_{i}$ & $0^{\circ}$ & $120^{\circ}$ & $240^{\circ}$ & $0^{\circ}$ & $120^{\circ}$ & $240^{\circ}$ \\
\hline$H_{i}$ & 210 & 210 & 210 & 210 & 210 & 210 \\
\hline \hline
\end{tabular}

Parameters that were not estimated.

TABLE 2 TIP index results and kinematic parameters. Relative velocity between the end-effector and the object in the tests is less than $1 \mathrm{~m} / \mathrm{s}$.

Index (mean 100 tests per Nominal Calibrated kind of movement)

TIP (fixed reference)

20.156

19.761

TIP (dynamic reference)

28.004

27.565

\section{CONCLUSIONS}

In this paper a calibration method was designed in order to improve accuracy of a parallel robot that is inspired in the Delta robot. The method was tested by means of a visual controller, and errors in the controller and the velocity of an object were used to obtain an index. Thus, both collections (nominal and corrected) of kinematic parameters were compared. Difference between parameters calibrated and non calibrated have strong influence in the performance of the error in the visual controller. Therefore performance of a tracking visual controller is improved although correction of the parameters is extremely small (errors in the controller are reduced more than 3\% when the robot is calibrated). Improvements in the controller performance can be explained as follows: Image visual controller makes use of the robot Jacobian and robot kinematic models (inverse and direct), when the controller corrects the robot trajectory, errors in the model have direct influence in the direction in which the controller tries to correct. On the other hand if the position of the object remains fixed, error in the visual controller converges to zero but the evolution of the error is different for two set of parameters. In this paper three main topics were discussed: 1.-Obtaining of a kinematic model based on incremental and measurements. 2.-Obtaining of a 3D measurement method from one camera in hand. 3.Obtaining of the influence of small kinematical errors in a classical visual controller.

Due to the influence of kinematic errors in the visual controller, future works are going to concentrate on studying 
if it is possible to directly calibrate the robot by minimizing the errors in the visual controller. Above approach brings out other problems as can be the addition of the time in the model. Future works include Ping-Pong playing, and some videos of the robot hitting a ball are shown in the web page:

http://www.disam.upm.es/vision/projects/robotenis/indexI $\underline{. h t m l}$

\section{ACKNOWLEDGMENT}

Authors thanks for financial support to the following institutions: Universidad Politécnica de Madrid from Madrid Spain; PROMEP and Universidad Autónoma de Querétaro from Queretaro, Mexico; and Universidad Nacional de San Juan from San Juan, Argentina.

\section{REFERENCES}

[1] F. Chaumette and S. Hutchinson, "Visual servo control. I. Basic approaches," IEEE Robotics \&amp;amp; Automation Magazine, vol. 13, Dec. 2006, pp. 82-90.

[2] G. Ecorchard and P. Maurine, "Self-calibration of delta parallel robots with elastic deformation compensation," 2005 IEEE/RSJ International Conference on Intelligent Robots and Systems, 2005.(IROS 2005), Ieee, 2005, p. $1283-1288$.

[3] A. Watanabe, S. Sakakibara, K. Ban, M. Yamada, G. Shen, and T. Arai, "A Kinematic Calibration Method for Industrial Robots Using Autonomous Visual Measurement," CIRP Annals - Manufacturing Technology, vol. 55, 2006, pp. 1-6.

[4] A. Nahvi, J.M. Hollerbach, and V. Hayward, "Calibration of a parallel robot using multiple kinematic closed loops," Proceedings of the 1994 IEEE International Conference on Robotics and Automation, IEEE Comput. Soc. Press, 1994, pp. 407-412.

[5] J. Ouyang, "Ball array calibration on a coordinate measuring machine using a gage block," Measurement, vol. 16, Dec. 1995, pp. 219-229.

[6] S. Bai and M.Y. Teo, "Kinematic calibration and pose measurement of a medical parallel manipulator by optical position sensors," Journal of Robotic Systems, 2003, pp. 201-209.

[7] Y. Zhang and F. Gao, "A calibration test of Stewart platform," 2007 IEEE International Conference on Networking, Sensing and Control, IEEE, 2007, pp. 297-301.

[8] M. Dinham and G. Fang, "A low cost hand-eye calibration method for arc welding robots," 2009 IEEE International Conference on Robotics and Biomimetics (ROBIO), IEEE, 2009, pp. 1889-1893.

[9] J. Sulzer and I. Kovač, "Enhancement of positioning accuracy of industrial robots with a reconfigurable fine-positioning module," Precision Engineering, vol. 34, Apr. 2010, pp. 201-217.

[10]G. Meng, L. Tiemin, and Y. Wensheng, "Calibration method and experiment of Stewart platform using a laser tracker," $S M C^{\prime} 03$ Conference Proceedings. 2003 IEEE International Conference on Systems, Man and Cybernetics. Conference Theme - System Security and Assurance (Cat. No.03CH37483), IEEE, 2003, pp. 2797-2802.

[11] A. Patel and K. Ehmann, "Calibration of a hexapod machine tool using a redundant leg," International Journal of Machine Tools and Manufacture, vol. 40, Mar. 2000, pp. 489-512.

[12]X.-D. Ren, Z.-R. Feng, and C.-P. Su, "A new calibration method for parallel kinematics machine tools using orientation constraint," International Journal of Machine Tools and Manufacture, vol. 49, Jul. 2009, pp. 708-721.

[13]N. Fazenda, E. Lubrano, S. Rossopoulos, and R. Clavel, "Calibration of the 6 DOF high-precision flexure parallel robot 'Sigma 6', Proceedings of 5th Parallel Kinematics Seminar. Chemnitz, Germany, 2006, p. 379-398.

[14] B. Karlsson and T. Brogårdh, "A new calibration method for industrial robots," Robotica, vol. 19, 2001, p. 691-693.

[15]O. Masory, "Kinematic calibration of a Stewart platform using pose measurements obtained by a single theodolite," Proceedings 1995
IEEE/RSJ International Conference on Intelligent Robots and Systems. Human Robot Interaction and Cooperative Robots, 1995, pp. 329-334.

[16]A. Pashkevich, D. Chablat, and P. Wenger, "Kinematic calibration of Orthoglide-type mechanisms from observation of parallel leg motions," Mechatronics, vol. 19, Jun. 2009, pp. 478-488.

[17] K. Großmann, B. Kauschinger, and S. Szatmári, "Kinematic calibration of a hexapod of simple design," Production Engineering, vol. 2, Jun. 2008, pp. 317-325.

[18]F. Y., Takeda; G., Shen; H., "A DBB-based kinematic calibration method for in-parallel actuated mechanisms using a Fourier series," Transactions- American Society Of Mechanical Engineers Journal Of Mechanical Design, vol. 126, 2004, pp. 856-865.

[19]H. Chanal, E. Duc, P. Ray, and J. Hascoet, "A new approach for the geometrical calibration of parallel kinematics machines tools based on the machining of a dedicated part," International Journal of Machine Tools and Manufacture, vol. 47, Jun. 2007, pp. 1151-1163.

[20]G. Canepa, J.M. Hollerbach, and A.J.M. a Boelen, "Kinematic calibration by means of a triaxial accelerometer," Proceedings of the 1994 IEEE International Conference on Robotics and Automation, IEEE Comput. Soc. Press, 1994, pp. 2776-2782.

[21]M. Jose Mauricio S.T., Motta; Guilherme C., De Carvalhob; R.S., "Robot calibration using a 3D vision-based measurement system with a single camera," Robotics and Computer-Integrated Manufacturing, 2001, pp. 487-497.

[22] Y. Meng and H. Zhuang, "Autonomous robot calibration using vision technology," Robotics and Computer-Integrated Manufacturing, vol. 23, 2007, pp. 436-446.

[23]N. Andreff and P. Martinet, "Vision-based self-calibration and control of parallel kinematic mechanisms without proprioceptive sensing," Intelligent Service Robotics, vol. 2, Mar. 2009, pp. 71-80.

[24]P. Renaud, N. Andreff, J.-M. Lavest, and M. Dhome, "Simplifying the kinematic calibration of parallel mechanisms using vision-based metrology," IEEE Transactions on robotics, vol. 22, Feb. 2006, p. 1222.

[25] A. M. Y., Amirat; J., Pontnau; F., "A three-dimensional measurement system for robot applications," Journal of Intelligent and Robotic Systems, vol. 9, 1994, pp. 291-299.

[26]Z. Zhang, "A flexible new technique for camera calibration," IEEE Transactions on Pattern Analysis and Machine Intelligence, vol. 22, 2000, pp. 1330-1334.

[27]R. Clavel, "Conception d'un robot parallele rapide a4 degrés de liberté," These de Doctorat, 1991.

[28]L.W. Tsai and R.E. Stamper, A parallel manipulator with only translational degrees of freedom, ISR; TR 1997-72, 1997.

[29] J. Sebastián, A. Traslosheros, L. Ángel, and F. Roberti, "Parallel Robot High Speed Object Tracking," Image Analysis and Recognition, Lecture Notes in Computer Science, M. Kamel and A. Campilho, eds., Berlin, Heidelberg: Springer Berlin Heidelberg, 2007, pp. 295-306.

[30] A. Traslosheros, L. Angel, R. Vaca, J.-M. Sebastián, F. Roberti, and R. Carelli, "New visual Servoing control strategies in tracking tasks using a PKM," Mechatronic Systems, A. Lazinica, ed., Vienna, Austria: InTech, 2010, pp. 117-146.

[31] S. Besnard and W. Khalil, "Identifiable parameters for parallel robots kinematic calibration," Proceedings 2001 ICRA. IEEE International Conference on Robotics and Automation (Cat. No.01CH37164), IEEE, 2001, pp. 2859-2866.

[32]A. Nahvi and J.M. Hollerbach, "The noise amplification index for optimal pose selection in robot calibration," IEEE INTERNATIONAL CONFERENCE ON ROBOTICS AND AUTOMATION, Citeseer, 1996, p. 647-654.

[33] J.-Y. Bouguet, "Camera calibration parameters," http://www.vision.caltech.edu/bouguetj/calib_doc/htmls/parameters.ht $m l, 2010$.

[34]L. Angel, A. Traslosheros, J. Sebastian, L. Pari, R. Carelli, and F. Roberti, "Vision-Based Control of the RoboTenis System," Lecture Notes In Control And Information Sciences, vol. 370, 2008, pp. 229240. 